\section{Cancer colorectaux métastatiques et thérapies ciblées anti-EGFR}

Frédéric Viret, Anthony Gonçalves

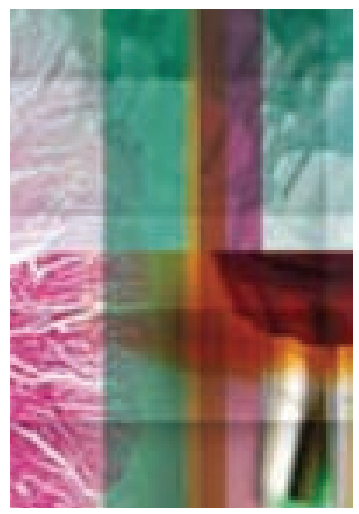

Département d'Oncolgie Médicale, Institut Paoli Calmettes-Marseille, Centre CRCM, Inserm, Unité 891, Université de la Méditerranée, 27, boulevard Leï Roure, 13009 Marseille, France. viretf@marseille.fnclcc.fr.

éventuellement la radiothérapie pour les cancers du rectum) et, pour les stades les moins précoces, une chimiothérapie adjuvante associant dérivés du $5 \mathrm{FU}$ et oxaliplatine [1].

Malgré ces traitements, et éventuellement dès le diagnostic, les CCR présentent une évolution métastatique. En tout, environ $50 \%$ à $60 \%$ des patients vont développer une récidive locale ou une métastase à distance avec une survie à 5 ans de moins de $5 \%$. À ce stade, les approches de résection chirurgicale peuvent conserver un objectif curatif, mais la grande majorité des patients sont candidats à des traitements médicaux systémiques. Globalement, la médiane de survie est inférieure ou égale à 9 mois en l'absence de traitement. Au cours des dix dernières années, la prise en charge des cancers colorectaux métastatiques a bénéficié de profonds changements permettant une amélioration sensible du pronostic : la démonstration de l'utilité d'une chimiothérapie, l'arrivée de nouvelles molécules comme l'oxaliplatine ou le CPT-11, l'utilisation de plusieurs lignes différentes et une meilleure coordination médico-chirurgicale permettant de plus nombreuses exérèses à visées curatrice de métastases.

Ainsi, au début des années 2000, trois essais utilisant le CPT-11 en première ligne de la maladie métastatique furent publiés. Une première étude [2] de phase III randomisée réalisée chez 683 patients a montré que le CPT-11 associé à 5FU/AF administrés en bolus 
présentait une efficacité supérieure par rapport au schéma $5 \mathrm{FU} / \mathrm{AF}$ de la «Mayo Clinic» en termes de taux de réponse (39\% versus $21 \%, p<0,001$ ), temps médian de progression ( 7 versus 4,3 mois, $p=0,004)$ et survie globale $(14,8$ versus 12,6 mois, $p=0,040)$. Une autre étude [3] de phase III utilisant le schéma infusionnel a comparé CPT- $11+5 \mathrm{FU}$ et acide folinique versus $5 \mathrm{FU}$ et acide folinique chez 387 patients. Deux schémas de 5 FU pouvaient être réalisés selon le choix de chaque centre : soit le schéma «de Gramont», soit le schéma $\mathrm{AIO}$. Les résultats montrent un meilleur taux de réponse ( $41 \%$ versus $23 \%, p<0,001$ ), un temps jusqu'à progression plus long $(6,7$ mois versus 4,4 mois, $p<0,001)$ et une médiane de survie plus longue $(17,4$ mois versus 14,1 mois, $p=0,03010$ ) dans le bras CPT- 11 $+5 \mathrm{FU}$ et acide folinique. Ces deux études ont permis l'utilisation du CPT-11 en première ligne thérapeutique dans le cancer colorectal métastatique aussi bien aux États-Unis (schéma IFL) qu'en Europe (schéma infusionnel).

Une étude de phase III, au même moment, a comparé l'oxaliplatine à la dose de $85 \mathrm{mg} / \mathrm{m}^{2}$ associé à un schéma LV5FU2 (Folfox4) à LV5FU2 seul, tous les 15 jours [4]. Au total, 420 patients ont été inclus et le taux de $\mathrm{R} 0$ rapporté était de $50,7 \%$ dans le bras à base d'oxaliplatine versus $22,3 \%$ dans le bras de référence $(p=0,0001)$. La survie médiane sans progression était de 9 mois versus 6,2 mois ( $p=0,0003)$. L'amélioration de la survie (16,2 versus 14,7 mois) n'était pas significative $(p=0,120)$. Par ailleurs, aux États-Unis, le schéma IFL a été comparé aux schémas Folfox4 et IROX (CPT-11 et oxaliplatine) dans une étude publiée en 2004 [5]. Les patients recevant le Folfox 4 avaient significativement un meilleur taux de réponse ( 45 versus $32 \%, p=0,002$ ), une meilleure survie médiane sans progression $(8,7$ mois versus 6,9 mois, $p=0,001)$ et une meilleure survie globale $(19,5$ mois versus 15 mois, $p=0,001)$ que ceux traités par le schéma IFL. À la suite de cette étude, la FDA (Food and Drug Administration) a autorisé l'utilisation du schéma Folfox en première ligne dans le traitement du cancer colorectal. Parallèlement, une étude européenne [6] a comparé le schéma Folfox au schéma Folfiri (CPT-11 et LV5FU2) chez des patients en première ligne. En cas de progression, un cross-over des schémas était effectué. Cette étude n'a pas retrouvé de différence entre les deux schémas que cela soit en termes de réponse ( $56 \%$ versus $54 \%$ ), de temps jusqu'à progression ( 8,5 mois versus 8 mois) ou de survie (20 mois versus 21,5 mois). Les survies médianes élevées obtenues dans cette étude pourraient être dues au fait que la plupart des patients étaient exposés aux trois drogues (5FU/LV, oxaliplatine et (PT-11) au cours de leurs parcours thérapeutique. Une récente méta-analyse étudiant l'ensemble des études de phase III utilisant ces trois drogues semble confirmer cette donnée [7]. Plus récemment, une simplification de l'administration du 5FU a été développée avec l'apparition de $5 \mathrm{FU}$ oral telle que la capecitabine. Ainsi, les schémas Xelox (oxaliplatine + capecitabine) et Xeliri (CPT-1l et capecitabine) ont été développés. Les principaux résultats d'une étude randomisée comparant les schémas Xelox et Folfox4 en première ligne de traitement du cancer colorectal métastatique sont disponibles depuis peu (Cassidy J, et al. ESMO 2006, abstract LBA3).
L'étude confirme la non-infériorité du schéma Xelox par rapport au schéma Folfox.

Très récemment, de nouvelles thérapeutiques moléculaires ciblées ont investi ce champ pathologique. Ces nouvelles thérapies ont pour cibles deux acteurs essentiels du phénotype tumoral : le VEGF, médiateur clé du phénomène de néo-angiogenèse et le récepteur de l'EGF (EGFR). Le seul agent anti-VEGF ayant démontré un avantage en terme de survie est le bevacizumab (Avastin ${ }^{\oplus}$ ), un anticorps monoclonal humanisé dirigé contre le VEGF. Trois essais démontrent son intérêt en association à la chimiothérapie. En association avec le schéma IFL, le bevacizumab augmente le taux de réponse de $35 \%$ à $45 \%$, le temps jusqu'à progression de 6 à 10 mois et la survie médiane de 15 à 20 mois avec un minimum de toxicité surajouté [8], dans une étude où cette association était comparée au schéma IFL seul en première ligne thérapeutique. Des résultats similaires semblent être obtenus avec des schémas à base d'oxaliplatine (Cassidy J, et al., ESM0 2006, abstract LA3). Enfin, un bénéfice de survie est également documenté lorsque le bevacizumab est utilisé en deuxième ligne thérapeutique après échec d'un schéma à base d'irinotecan, associé au Folfox [9]. Les approches ciblant I'EGFR sont développées dans les deux paragraphes suivants.

\section{EGFR et ciblage thérapeutique}

Le récepteur de l'EGF (Epidermal Growth Factor Receptor, HERl, $\varepsilon R B B 1, \varepsilon G F R$ ) est une glycoprotéine transmembranaire de $170 \mathrm{kDa}$, chef de file de la famille des récepteurs ERBB (qui comprend également $\varepsilon R B B 2, \varepsilon R B B 3$ et $\varepsilon R B B 4$ ). II comprend un domaine extracellulaire impliqué dans la reconnaissance et la liaison des ligands spécifiques, un domaine transmembranaire, notamment impliqué dans les interactions entre récepteurs, et un domaine intracytoplasmique qui contient l'activité enzymatique tyrosine kinase [10]. En réponse à la stimulation par un ligand spécifique tel que l'EGF ou le TGF $\alpha$ (transforming growth factor $\alpha$ ), le récepteur s'homodimérise ou s'hétérodimérise avec les autres membres de la famille. Le domaine extracellulaire est composé de 4 domaines, 2 domaines $L$ et 2 domaines riches en cystéine (CR). Ces domaines sont organisés selon la séquence suivante de l'extrémité $\mathrm{N}$ - vers C-terminale: domaine I à $4=\mathrm{Ll}$ CR1-L2-CR2. Les domaines interagissant directement avec le ligand sont les domaines I (L1) et III (L2). Le résultat de cette interaction est le démasquage du domaine II (CRI), séquestré à l'état inactif au sein 
du domaine IV (CR2), qui permet le phénomène de dimérisation. Cette dimérisation entraîne une phosphorylation des résidus tyrosine du domaine kinase intracytoplasmique des récepteurs engagés, à l'origine de l'initiation d'une cascade de signalisation complexe. Ces voies de signalisation comprennent notamment la voie de prolifération Ras-Raf-MAPK, la voie anti-apoptotique phosphatidylinositol-3 kinase (PI3K)-Akt, ainsi que les voies SAPK (stressactivated protein kinase) et STAT (signal transducers and activators of transcription). En pathologie, une dérégulation de l'activité de I'EGFR aboutit à favoriser les phénomènes de prolifération cellulaire, d'échappement à la mort cellulaire programmée, de néo-angiogenèse, de motilité cellulaire, de réparation de I'ADN, d'invasion tissulaire et de métastases et constitue donc un événement très favorable à l'acquisition et au maintien du phénotype cancéreux. Ainsi, une surexpression de l'EGFR a été documentée dans de nombreuses néoplasies épithéliales, incluant les CCR ( $25 \%$ à $77 \%$ des cas selon les études et les techniques utilisées) [11]. Dans les CCR, cette surexpression a pu être associée à un pronostic défavorable, même si les données sont controversées [12-16]. Les mécanismes qui conduisent à une dérégulation de l'activité de l'EGFR dans les néoplasies épithéliales ne sont que partiellement connus et très probablement multiples: boucle autocrine ligand-récepteur et/ou altérations génomique (amplification, surexpression, mutations, délétions, réarrangements) induisant une activation constitutionnelle de la voie. Un certain nombre de mutations de l'EGFR modifiant la fonction ou l'activation du récepteur ont été mises en évidence dans différentes pathologies tumorales, notamment dans les glioblastomes [17] avec la mutation EGFRvIII qui concerne le domaine extracellulaire et, plus récemment, dans certains adénocarcinomes pulmonaires [18-20] avec des mutations concernant le domaine à activité tyrosine kinase de la molécule dans sa portion intracytoplasmique. Ces dernières mutations semblent absentes ou très rares dans les CCR.

Les tentatives de modulation thérapeutique de l'EGFR ont été initiées il y a plus de 20 ans et constituent les toutes premières approches démontrant un effet antiprolifératif par ciblage d'un récepteur à activité tyrosine kinase [22, 23]. À l'heure actuelle, il existe deux catégories principales d'agents anti-EGFR exploités en clinique : les petites molécules inhibitrices du domaine tyrosine kinase intracytoplasmique du récepteur (gefitinib, Iressa ${ }^{\circledR}$; erlotinib, Tarceva ${ }^{\circledR}$, TKI) et les anticorps monoclonaux dirigés contre le domaine extracellulaire, tels que le cétuximab (Erbitux ${ }^{\circledR}$ ) ou plus récemment le panitumumab (Vectibix ${ }^{\circledR}$ ) (ACM). Ainsi, le cetuximab, anticorps chimérique $\operatorname{lgGl}$, se fixe sur la partie extracellulaire du récepteur avec une haute affinité $(K d=0,39 \mathrm{nmol} / \mathrm{l})$ et s'oppose de façon compétitive à la liaison des ligands spécifiques bloquant ainsi l'activation du récepteur. Sur le plan structural, le cetuximab interagit avec le domaine III de l'ectodomaine, empêchant la fixation du ligand à ce niveau. Même si la liaison du ligand au domaine I n'est pas abolie par l'anticorps, l'encombrement du domaine III a pour conséquence de diminuer significativement les modifications de conformation structurales nécessaire au phénomène de dimérisation [24]. Par ailleurs, le cetuximab induit une internalisation du récepteur, suivie d'une dégradation [25]. Les petites molécules, elles, inhibent de façon compétitive la liaison de l'ATP au niveau du domaine porteur de l'activité enzymatique tyrosine kinase, induisant l'abrogation de celle-ci. Les effets biologiques du blocage de l'EGFR et des voies de signalisation qui en dépendent, que ce soit par les anticorps monoclonaux ou par les petites molécules comprennent: l'arrêt du cycle cellulaire en phase Gl, du fait de l'accumulation de le protéine p2 $7^{\text {kipl }}$, inhibiteur de CDK2, qui aboutit à l'hypophosphorylation de la protéine $\mathrm{Rb}$ [26] ; potentialisation de l'apoptose [27, 28] ; inhibition de l'angiogenèse $[29,30]$; inhibition de l'invasion tumorale et du phénomène métastatique $[30,31]$; potentialisation des effets de la chimiothérapie et de la radiothérapie [32-35]. Les anticorps monoclonaux peuvent de plus initier une réponse immunologique de type ADCC (antibody-dependent cellular cytotoxicity) [36]. En pratique clinique, les inhibiteurs de l'EGFR ont montré une activité dans un certain nombre de tumeurs solides épithéliales. À l'heure actuelle, le

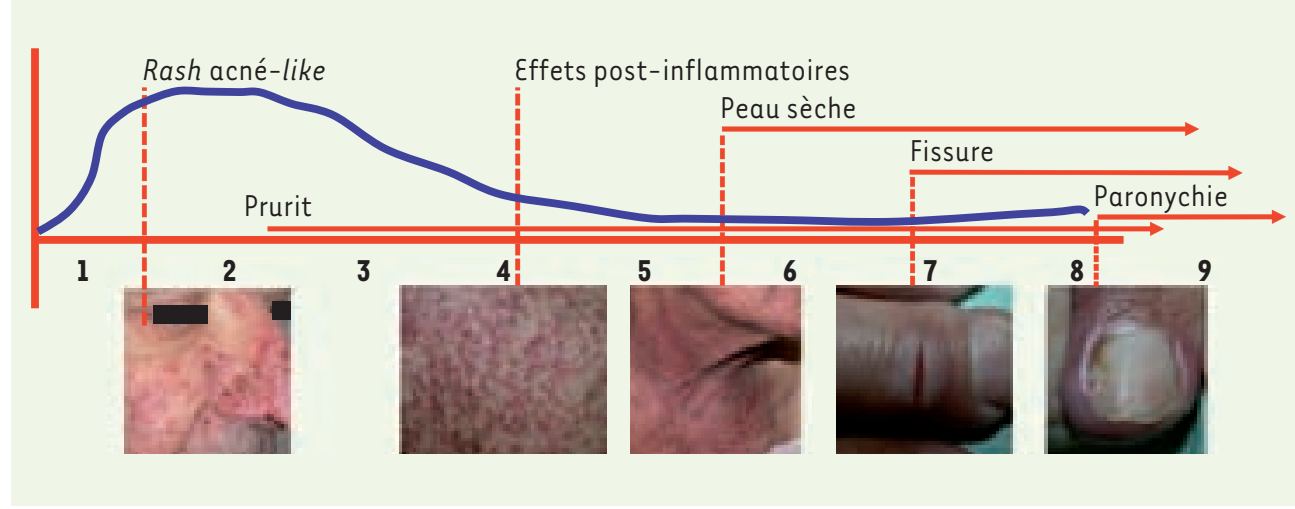

Figure 1. Effets secondaires induits par les inhibiteurs de l'EGFR. Les différentes manifestations sont décrites et enregistrées chez chaque patient traité selon des critères de gravité sur une échelle de 1 à 9 . Une réaction majeure peut entraîner la diminution des doses, voire l'arrêt du traitement, bien qu'elle soit généralement corrélée à une efficacité thérapeutique sur le cancer lui-même. 
seul ACM anti-EGFR disposant d'une AMM est le cetuximab, dans les CCR métastatiques et, plus récemment, dans les cancers de la sphère ORL localisés, en association avec la chimiothérapie (CCR) ou la radiothérapie (ORL). Les $T K I$ sont actuellement seulement indiqués dans les cancers pulmonaires non à petites cellules de stade avancé, en échec de chimiothérapie.

\section{Place du Cetuximab et des inhibiteurs de l'EGFR dans les CCR}

Dans les études précoces de phase I/II, le cetuximab a démontré un profil de tolérance tout à fait acceptable, incluant essentiellement une toxicité cutanée très fréquente de type rash cutané acnéiforme et plus rarement des réactions anaphylactoïdes (Figure l).

Ces réactions allergiques peuvent être sévères dans moins de $2 \%$ des cas et apparaissent dans les premières minutes de la perfusion initiale [37], répondant généralement bien aux traitements symptomatiques. Des anticorps humains anti-souris ont été notés dans $4 \%$ des cas, sans lien apparent avec les réactions allergiques cliniques, et sans conséquences évidentes sur la pharmacocinétique du cetuximab, compatible avec une administration hebdomadaire. La dose optimale biologique, déterminée comme la dose saturante des mécanismes de clairance de l'anticorps, est située entre 200 et $400 \mathrm{mg} / \mathrm{m}^{2}$, et ces posologies ont été identifiées comme suffisantes pour inhiber l'activation du récepteur ainsi que les voies d'aval [38].

Dans les CCR métastatiques, le cetuximab a démontré son efficacité chez des patients réfractaires, seul ou en association à l'irinotecan. Un essai clinique de phase II a évalué l'activité du cetuximab dans une population de 57 patients présentant un CCR métastatique résistant à l'irinotecan, retrouvant un taux de réponse de l'ordre de $11 \%$ avec $35 \%$ de maladie stable [39] et une médiane de survie de 6,4 mois. Plus récemment, des résultats très similaires ont été retrouvés pour le cetuximab en monothérapie chez des patients résistant à l'irinotecan et à l'oxaliplatine [40]: $8 \%$ à $12 \%$ de réponses objectives et une médiane de survie globale de l'ordre de 6 mois. En association, Saltz et al. ont initialement décrit un taux de réponse de l'ordre de $20 \%$ avec $27 \%$ de maladie stable pour le cetuximab combiné à l'irinotecan chez 121 patients résistant à cette dernière molécule. Dans l'essai d'association de référence (essai BOND), l'efficacité et la sécurité du schéma cetuximab/irinotecan étaient comparées à celles du cetuximab seul chez 329 patients présentant un CCR métastatique réfractaire à l'irinotecan. Le taux de réponse de l'association par rapport au cetuximab seul était de 22,9\% (95\% Cl, 17,5-29,1\%) contre $10,8 \%(95 \% \mathrm{Cl}, 5,7-18,1 \%)(p=0,007)$, le temps jusqu'à progression médian était de 4,1 mois contre 1,5 mois $(p<0,0001)$, le taux de contrôle de la maladie était de $56 \%$ versus $32 \%$ et la survie médiane était de 8,6 mois contre 6,9 mois $(p=0,48)$, avec des toxicités supérieures dans le bras d'association, mais non différentes de celles attendues avec l'irinotecan seul [41]. Cette étude a permis l'enregistrement du cetuximab chez des patients réfractaires à une chimiothérapie à base d'irinotecan.
Il faut noter qu'un autre ACM anti-EGFR, le panitumumab (Vectibix ${ }^{\oplus}$ ), un anticorps entièrement humanisé de haute affinité pour l'EGFR, avec le même profil de tolérance, excepté les réactions allergiques, a démontré des résultats comparables en monothérapie chez des patients résistant à l'oxaliplatine et/ou à l'irinotecan (Hecht et al., ASCO 2004): $10 \%$ de réponse et $38 \%$ de maladies stables, la durée médiane de réponse était de 5,2 mois ( $95 \% \mathrm{Cl}, 4,5-7,5$ mois), la médiane de survie sans progression était de 2,0 mois ( $95 \% \mathrm{Cl}, 1,9-3,8$ mois), et la survie globale médiane était de 7,9 mois ( $95 \% \mathrm{Cl}, 5,7-9,9$ mois). Très récemment, cet $A C M$ en monothérapie a démontré un avantage en terme de survie sans progression chez des patients résistant à l'oxaliplatine et l'irinotecan, dans un essai randomisé contre soins de support optimaux, conduisant à son enregistrement dans cette indication par la FDA. II faut noter que l'ensemble de ces études, avec cetuximab et panitumumab, ont été réalisées chez des patients présentant une tumeur EGFR positive en immunohistochimie.

À l'heure actuelle, de nombreuses études sont récemment complétées ou encore en cours évaluant le cetuximab ou le panitumumab dans d'autres schémas d'association [42] et/ou à des stades plus précoces, en association avec les différentes chimiothérapies et/ou le bevacizumab, en première ligne métastatique avec des résultats prometteurs [43]. Des essais sont également cours en situation adjuvante ou néoadjuvante [44].

Les petites molécules inhibitrices de type TKI (gefitinib, erlotinib) ciblant l'EGFR ont également été explorées dans les CCR avancés ou métastatiques [45-52]. Dans la plupart des études, l'expression d'EGFR n'était pas un pré-requis à l'inclusion. De façon générale, ces molécules semblaient peu ou pas actives administrées en monothérapie, leur utilisation en association à la chimiothérapie et/ou la radiothérapie pouvant être limitée par la toxicité digestive, et notamment la diarrhée, même si des résultats intéressants en terme d'activité ont pu être suggérés.

\section{Cetuximab et paramètres moléculaires prédictifs potentiels: impact des mutations de KRAS}

De nombreuses études rétrospectives ont été réalisées à la recherche de facteurs prédictifs de la réponse thérapeutique, permettant d'identifier des sous-groupes de patients à plus ou moins haute probabilité de réponse à un traitement comportant un anticorps monoclonal ciblant l'EGFR. Comme 
précédemment souligné, le développement du cetuximab s'est quasi-exclusivement réalisé chez des patients exprimant l'EGFR en immunohistochimie. Pourtant, aucun des essais majeurs dans le développement clinique du cetuximab n'a permis de mettre évidence un lien entre le niveau d'expression de I'EGFR et l'activité du médicament $[39,41]$. De façon encore plus troublante, il a été retrouvé un taux de réponse objective de l'ordre de $25 \%$ dans des populations de patients dont la tumeur était négative pour l'EGFR et qui recevait un traitement

\begin{tabular}{|c|c|c|c|}
\hline & Répondeurs & $\begin{array}{l}\text { Non } \\
\text { répondeurs }\end{array}$ & $\begin{array}{c}\text { Valeurs de p (test } \\
\text { exact de Fisher ou } \chi^{2} \text { ) }\end{array}$ \\
\hline \multicolumn{4}{|l|}{ Moroni et al. } \\
\hline Mutation KRAS & 2 & 8 & 0,428 \\
\hline KRAS WT & 8 & 13 & \\
\hline \multicolumn{4}{|l|}{ Lievre et al. } \\
\hline Mutation KRAS & 0 & 13 & 0,0003 \\
\hline KRAS WT & 11 & 6 & \\
\hline \multicolumn{4}{|l|}{ Di Fiore et al. } \\
\hline Mutation KRAS & 0 & 22 & 0,002 \\
\hline KRAS WT & 12 & 25 & \\
\hline \multicolumn{4}{|l|}{ Khambata-Ford et al. } \\
\hline Mutation KRAS & 3 & 27 & 0,0001 \\
\hline KRAS WT & 24 & 26 & \\
\hline \multicolumn{4}{|l|}{ Benvenuti et al.* } \\
\hline Mutation KRAS & 1 & 21 & 0,006 \\
\hline KRAS WT & 10 & 16 & \\
\hline \multicolumn{4}{|l|}{ Frattini et al. } \\
\hline Mutation KRAS & 1 & 9 & 0,04 \\
\hline KRAS WT & 9 & 8 & \\
\hline \multicolumn{4}{|l|}{ De Roocke et al. } \\
\hline Mutation KRAS & 0 & 42 & $1,3310-7$ \\
\hline KRAS WT & 27 & 39 & \\
\hline \multicolumn{4}{|l|}{ Global } \\
\hline Mutation KRAS & 7 & 142 & $9,10-16$ \\
\hline KRAS WT & 101 & 133 & \\
\hline
\end{tabular}

Tableau I. Mutation activatrice de l'axe RAS/RAF et réponse aux traitements de type anticorps monoclonaux ciblant l'EGFR. *Mutation de KRAS ou BRAF. à base de cetuximab, taux similaire à celui obtenu dans des populations EGFR+ (Lentz et al., ASCO 2004) [53, 54]. La survenue d'une toxicité cutanée, un effet secondaire typique du cetuximab comme des autres anti-EGFR qu'ils soient TKI ou ACM, a été retrouvée comme associée à une plus forte probabilité de réponse et de survie dans les CCR traités par cetuximab, comme dans d'autres localisations tumorales traitées par d'autres types de molécules anti-EGFR [41]. Cependant, cette réaction cutanée s'apparente plus à un «surrogate» d'un effet biologique sur l'EGFR au moins cutané, qu'à un véritable marqueur prédictif. Parmi les paramètres potentiellement impliqués dans les mécanismes de résistance ou de sensibilité au cetuximab, ont été rapportés ces derniers mois: le nombre de copies de l'EGFR évalué en FISH, avec la mise évidence d'une activité anti-tumorale plus marquée en cas d'amplification ou de polysomie [55, 56], l'expression des ligands de l'EGFR, tels que l'amphiréguline et l'épiréguline [57] associés à une plus grande probabilité de survie sans progression, l'expression immunohistochimique de PTEN, dont le faible niveau serait associé à la résistance thérapeutique, certains polymorphismes du récepteur Fc gamma des immunoglobuline $G$ et surtout le statut mutationnel de l'axe KRAS/BRAF.

KRAS est une petite protéine $G$ qui joue un rôle majeur dans la voie de signalisation RAF/MAPK, l'une des voies de prolifération majeure recrutée par les récepteurs à activité tyrosine kinase, notamment la voie de l'धGFR. Comme éléments centraux dans la voie des MAPK, les différentes isoformes de RAF (ARAF, BRAF et CRAF/RAF-1) sont impliquées dans la régulation de la prolifération, mais également de la différenciation, la survie cellulaire, l'angiogenèse, la migration et l'adhésion. II s'agit d'une famille de sérine/ thréonine kinases cytosoliques, situées en aval de KRAS et en amont de la kinase MEK. Que ce soit directement ou indirectement, l'activation de KRAS conduit au recrutement de ces protéines à la membrane plasmique et à leur activation. Ces différentes isoformes, et notamment BRAF qui est le plus puissant, conduisent à l'activation (c'est-à-dire à la phosphorylation) de 
MEK, qui phosphoryle ERK. ERK phosphorylé conduit à la régulation d'un très grand nombre de substrats cytosoliques et/ou nucléaires, notamment S6 kinase, Elk-1, ARF, p27 Kipl, etc. II existe également des effets biologiques de RAF qui semblent indépendants de la voie $M \varepsilon K / \varepsilon R K$, et qui concernent notamment des processus de régulation de l'apoptose dans sa voie intrinsèque mitochondriale, avec un effet plutôt anti-apoptotique.

Des mutations activatrices du gène KRAS ont été mises en évidence dans environ $35 \%$ des cancers colorectaux, et sont considérés comme un événement précoce dans l'oncogenèse colorectale [21]. Les mutations concernent l'exon 2 (codon 12 notamment) et sont également associées à un pronostic défavorable de façon intrinsèque. Parallèlement, des mutations activatrices de BRAF, concernant essentiellement l'exon 15 (V600E), ont été mises en évidence dans environ $10 \%$ des cancers colorectaux et semblent associées à un mauvais pronostic global, notamment dans un contexte non MSI. Comme les mutations de KRAS, les mutations activatrices de BRAF sont associées à une activité oncogénique, et notamment à une hyperactivation de la voie MAPK.

Très clairement, plusieurs études récentes et convergentes ont montré que l'existence d'une mutation de l'exon 1 de KRAS était associée à une probabilité de réponse à un traitement à base de cetuximab ou de panitumumab, faible voire nulle pour certains. Ainsi, la première étude identifiant ce facteur défavorable portait sur 30 patients traités par cetuximab, 11 patients répondeurs et 19 patients en maladie stable ou progressive [58]. Aucune mutation n'était présente parmi les 11 patients répondeurs $(0 \%,[95 \% \mathrm{Cl}, 0-28,5 \%])$, tandis que 13 des 19 tumeurs non répondeuses présentaient une mutation de I'exon 1 de $\operatorname{KRAS}(68,4 \%$, [95\% Cl, 43,5-87,5\%], p =0,0003). La survie globale médiane des patients présentant une mutation était par ailleurs significativement plus faible. Une deuxième étude portant sur 59 patients présentant un CCR métastatique réfractaire, recevant un traitement à base de cetuximab, a confirmé ces données, ne retrouvant aucune mutation de KRAS parmi les 12 patients présentant une réponse objective, avec un temps jusqu'à progression significativement raccourci chez les patients présentant une mutation [59]. Une troisième étude a étendu ces résultats aux mutations de l'axe RAS/RAF, en intégrant les mutations activatrices de BRAF (V600E) [60]. D'autres études encore plus récentes vont dans le même sens, celui d'un impact très défavorable de l'existence d'une mutation activatrice de l'axe RAS/RAF quant à la probabilité de réponse aux traitements de type anticorps monoclonaux ciblant l'EGFR. Ces données sont regroupées au sein du tableau récapitulatif (Tableau I) et leur analyse «poolée » est très évocatrice de ce point de vue.

En situation de première ligne métastatique, une étude randomisée a comparé un schéma Folfiri au schéma Folfiri + cetuximab [61]. L'analyse, rétrospective, démontre clairement un bénéfice en termes de réponse et de survie sans progression pour les patients KRAS WT qui reçoivent I'association Folfiri-cetuximab. Des résultats similaires ont été obtenus lorsque le schéma Folfox remplace le schéma Folfiri [62] : seuls les patients KRAS WT tirent un béné- fice de l'association Folfox + cetuximab. À l'inverse, l'association Folfox + cetuximab semble délétère en terme de PFS pour les patients KRAS mutés ( 5,5 versus $8,8$ mois, $p=0,0192, H R=1,83)$. Une autre étude, de phase I/II, évaluant l'efficacité d'une escalade de doses du cetuximab chez des patients atteints de CCM évoluant après un traitement à base d'irinotecan et ne présentant pas de toxicité cutanée, a retrouvé des résultats identiques [63].

Des résultats préliminaires de l'association cetuximab-bevacizumab + Folfiri ou Folfox ont été rendus publics au début de l'année 2008. Alors qu'une étude de phase [64] avait retrouvé des résultats prometteurs de l'association cetuximab-bevacizumab-CPT11, deux études ne retrouvent pas de bénéfices en termes de réponses ou de PFS $[65,66]$. Une analyse plus mature ainsi qu'une étude des sous-groupes, notamment en fonction du statut KRAS, est attendue. Ces études confirment la valeur prédictive et peutêtre pronostique des mutations du gène KRAS en situation métastatique lorsqu'une thérapie antiEGFR est utilisée. Une confirmation de ces résultats de façon prospective est nécessaire. Ces résultats posent également un certain nombre de questions: Pourquoi une réponse au traitement n'est pas observée chez l'ensemble des patients KRAS WT? Quel traitement proposer aux patients ayant des tumeurs KRAS mutées? Pourquoi l'association de bevacizumab et d'un anti-EGR (cetuximab ou panitumumab) ne fournit pas les résultats escomptés? $\diamond$

\section{SUMMARY}

Metastatic colorectal cancers

and targeted therapy against EGFR

Colorectal cancer (CRC) (CRC) is one of the leading causes of mortality and accounts for approximately 200000 deaths per year in Europe and the USA. Chemotherapy and radiotherapy have established roles in the treatment of colorectal cancer and can contribute to cure rate, prolongation of survival, reduction of local rates of recurrence and enhanced quality of life in patients with advanced disease. However, over the past few years there have been major advances in our understanding of the molecular basis of this tumour and its progression from adenoma to carcinoma that hold potential for translation into novel strategies for the treatment of CRC. Furthermore, newer agents directed against different intracellular targets have also been shown to be efficacious in CRC treatment. Such improvements should continue to lead to increased cure rates in early disease and better overall survival in advanced cases. $\diamond$ 


\section{RÉFÉRENCES}

1. Andre T, Boni C, Mounedji-Boudiaf L, et al. Oxaliplatin, fluorouracil, and leucovorin as adjuvant treatment for colon cancer. N EnglJ Med 2004 ; $350: 2343-51$.

2. Saltz LB, Cox JV, Blanke C, et al. Irinotecan plus fluorouracil and leucovorin for metastatic colorectal cancer. Irinotecan study group. $N$ Engl J Med 2000 ; 343 : 905-14.

3. Douillard JY, Cunningham D, Roth AD, et al. Irinotecan combined with fluorouracil compared with fluorouracil alone as first-line treatment for metastatic colorectal cancer: a multicentre randomised trial. Lancet $2000 ; 355: 1041-7$

4. De Gramont A, Figer A, Seymour M, et al. Leucovorin and fluorouracil with or without oxaliplatin as first-line treatment in advanced colorectal cancer.J Clin Oncol 2000; 18 : 2938-47.

5. Goldberg RM, Sargent DJ, Morton RF, et al. A randomized controlled trial of fluorouracil plus leucovorin, irinotecan, and oxaliplatin combinations in patients with previously untreated metastatic colorectal cancer. J Clin Oncol 2004; $22: 23-30$.

6. Tournigand C, Andre T, Achille $\varepsilon$, et al. FOLFIRI followed by FOLFOX6 or the reverse sequence in advanced colorectal cancer: a randomized GERCOR study. J Clin Oncol $2004 ; 22$ : 229-37.

7. Grothey A, Sargent D, Goldberg RM, Schmoll HJ. Survival of patients with advanced colorectal cancer improves with the availability of fluorouracil-leucovorin, irinotecan, and oxaliplatin in the course of treatment. J Clin Oncol 2004 ; 22 : 1209-14.

8. Hurwitz H, Fehrenbacher L, Novotny W, et al. Bevacizumab plus irinotecan, fluorouracil, and leucovorin for metastatic colorectal cancer. N Engl I Med 2004 ; 350 : 2335-42.

9. Giantonio BJ. Bevacizumab in the treatment of metastatic colorectal cancer (mCRC) in second- and third-line settings. Semin Oncol 2006; 33 (suppl 10) : S15-8.

10. Ullrich A, Schlessinger J. Signal transduction by receptors with tyrosine kinase activity. Cell $1990 ; 61: 203-12$.

11. Grandis JR, Sok JC. Signaling through the epidermal growth factor receptor during the development of malignancy. Pharmacol Ther 2004 ; $102: 37-46$.

12. Spano JP, Lagorce C, Atlan D, et al. Impact of EGFR expression on colorectal cancer patient prognosis and survival. Ann Oncol 2005 $16: 102-8$.

13. Lee JC, Wang ST, Chow NH, Yang HB. Investigation of the prognostic value of coexpressed erbB family members for the survival of colorectal cancer patients after curative surgery. Eur J Cancer $2002 ; 38$ : 1065-71.

14. McKay JA, Murray LJ, Curran S, et al. Evaluation of the epidermal growth factor receptor (EGFR) in colorectal tumours and lymph node metastases. Eur J Cancer 2002 ; $38: 2258-64$.

15. Kopp R, Rothbauer $\varepsilon$, Mueller $\varepsilon$, et al. Reduced survival of rectal cancer patients with increased tumor epidermal growth factor receptor levels. Dis Colon Rectum $2003 ; 46: 1391-9$.

16. Galizia G, Lieto $\varepsilon$, Ferraraccio F, et al. Prognostic significance of epidermal growth factor receptor expression in colon cancer patients undergoing curative surgery. Ann Surg Oncol $2006 ; 13: 823-35$.

17. Kuan CT, Wikstrand CJ, Bigner DD. EGF mutant receptor vIII as a molecular target in cancer therapy. Endocr Relat Cancer $2001 ; 8: 83-96$.

18. Lynch TJ, Bell DW, Sordella R, et al. Activating mutations in the epidermal growth factor receptor underlying responsiveness of non-small-cell lung cancer to gefitinib. N Engl J Med 2004 ; $350: 2129$-39.

19. Paez JG, Janne PA, Lee JC, et al. EGFR mutations in lung cancer: correlation with clinical response to gefitinib therapy. Science 2004 ; $304: 1497-500$.

20. Pao W, Miller V, Zakowski M, et al. EGF receptor gene mutations are common in lung cancers from never smokers and are associated with sensitivity of tumors to gefitinib and erlotinib. Proc Natl Acad Sci USA 2004 ; 101 : 13306-11.

21. Barber TD, Vogelstein B, Kinzler KW, Velculescu VE. Somatic mutations of EGFR in colorectal cancers and glioblastomas. $N$ Engl J Med 2004 ; $351: 28-83$.

22. Sato JD, Kawamoto $T$, Le $A D$, et al. Biological effects in vitro of monoclonal antibodies to human epidermal growth factor receptors. Mol Biol Med 1983; 1:511-29.

23. Kawamoto T, Sato JD, Le A, et al. Growth stimulation of A431 cells by epidermal growth factor: identification of high-affinity receptors for epidermal growth factor by an anti-receptor monoclonal antibody. Proc Natl Acad Sci USA $1983 ; 80: 1337-41$.

24. Aivado M, Spentzos D, Alterovitz G, et al. Optimization and evaluation of surface-enhanced laser desorption/ionization time-of-flight mass spectrometry (SELDI-TOF MS) with reversedphase protein arrays for protein profiling. Clin Chem Lab Med 2005 ; 43 : 133-40.

25. Fan Z, Lu Y, Wu X, Mendelsohn J. Antibody-induced epidermal growth factor receptor dimerization mediates inhibition of autocrine proliferation of A431 squamous carcinoma cells. J Biol Chem $1994 ; 269: 27595-602$.

26. Peng D, Fan Z, Lu Y, et al. Anti-epidermal growth factor receptor monoclonal antibody 225 up-regulates $\mathrm{p} 27^{\mathrm{KIPl}}$ and induces $\mathrm{Gl}$ arrest in prostatic cancer cell line DU145. Cancer Res $1996 ; 56: 3666-9$.

27. Wu X, Fan Z, Masui H, et al. Apoptosis induced by an anti-epidermal growth factor receptor monoclonal antibody in a human colorectal carcinoma cell line and its delay by insulin. J Clin Invest $1995 ; 95: 1897-905$.

28. Liu B, Fang M, Schmidt M, et al. Induction of apoptosis and activation of the caspase cascade by anti-EGF receptor monoclonal antibodies in DiFi human colon cancer cells do not involve the c-jun $\mathrm{N}$-terminal kinase activity. BrJ Cancer $2000 ; 82: 1991-9$.

29. Vincenzi $B$, Santini $D$, Russo A, et al. Angiogenesis modifications related with cetuximab plus irinotecan as anticancer treatment in advanced colorectal cancer patients. Ann Oncol 2006 ; $17: 835-41$.

30. Perrotte P, Matsumoto T, Inoue K, et al. Anti-epidermal growth factor receptor antibody C225 inhibits angiogenesis in human transitional cell carcinoma growing orthotopically in nude mice. Clin Cancer Res $1999 ; 5: 257-64$.

31. 0-Charoenrat P, Modjtahedi H, Rhys-Evans P, et al. Epidermal growth factor-like ligands differentially up-regulate matrix metalloproteinase 9 in head and neck squamous carcinoma cells. Cancer Res $2000 ; 60: 1121-8$.

32. Aboud-Pirak $\varepsilon$, Hurwitz $\varepsilon$, Pirak ME, et al. Efficacy of antibodies to epidermal growth factor receptor against KB carcinoma in vitro and in nude mice. J Natl Cancer Inst 1988 ; $80: 1605-11$.

33. Baselga J, Norton L, Masui $\mathrm{H}$, et al. Antitumor effects of doxorubicin in combination with anti-epidermal growth factor receptor monoclonal antibodies. J Natl Cancer Inst 1993 ; $85: 1327-33$.

34. Fan Z, Baselga J, Masui H, Mendelsohn J. Antitumor effect of anti-epidermal growth factor receptor monoclonal antibodies plus cis-diamminedichloroplatinum on well established A431 cell xenografts. Cancer Res $1993 ; 53: 4637-42$.

35. Prewett MC, Hooper AT, Bassi R, et al. Enhanced antitumor activity of anti-epidermal growth factor receptor monoclonal antibody IMC-C225 in combination with irinotecan (CPT-11) against human colorectal tumor xenografts. Clin Cancer Res 2002 ; $8: 994-1003$

36. Naramura M, Gillies SD, Mendelsohn J, et al. Therapeutic potential of chimeric and murine anti-(epidermal growth factor receptor) antibodies in a metastasis model for human melanoma. Cancer Immunol Immunother $1993 ; 37$ : 343-9.

37. Baselga J, Pfister D, Cooper MR, et al. Phase I studies of anti-epidermal growth factor receptor chimeric antibody C225 alone and in combination with cisplatin. J Clin Oncol 2000 ; 18: 904-14.

38. Shin DM, Donato NJ, Perez-Soler R, et al. Epidermal growth factor receptor-targeted therapy with C225 and cisplatin in patients with head and neck cancer. Clin Cancer Res 2001 ; 7: 1204-13.

39. Saltz LB, Meropol NJ, Loehrer PJ Sr, et al. Phase II trial of cetuximab in patients with refractory colorectal cancer that expresses the epidermal growth factor receptor. J Clin Oncol 2004 ; 22: 1201-8.

40. Lenz HJ, Van Cutsem $\varepsilon$, Khambata-Ford S, et al. Multicenter phase II and translational study of cetuximab in metastatic colorectal carcinoma refractory to irinotecan, oxaliplatin, and fluoropyrimidines. J Clin Oncol $2006 ; 24: 4914-21$.

41. Cunningham $D$, Humblet $Y$, Siena $S$, et al. Cetuximab monotherapy and cetuximab plus irinotecan in irinotecan-refractory metastatic colorectal cancer. N Engl J Med 2004 $351: 337-45$.

42. Souglakos J, Kalykaki A, Vamvakas L, et al. Phase II trial of capecitabine and oxaliplatin (CAPOX) plus cetuximab in patients with metastatic colorectal cancer who progressed after oxaliplatin-based chemotherapy. Ann Oncol $2006 ; 18: 305-10$.

43. Folprecht $G$, Lutz MP, Schoffski $P$, et al. Cetuximab and irinotecan/5-fluorouracil/folinic acid is a safe combination for the first-line treatment of patients with epidermal growth factor receptor expressing metastatic colorectal carcinoma. Ann Oncol $2006 ; 17: 450-6$.

44. Van Cutsem $\varepsilon$. Challenges in the use of epidermal growth factor receptor inhibitors in colorectal cancer. Oncologist $2006 ; 11: 1010-7$.

45. Daneshmand M, Parolin DAE, Hirte HW, et al. A pharmacodynamic study of the epiderma growth factor receptor tyrosine kinase inhibitor ZD1839 in metastatic colorectal cancer patients. Clin Cancer Res $2003 ; 9: 2457-64$.

46. Cho CD, Fisher GA, Halsey J, Sikic BI. Phase I study of gefitinib, oxaliplatin, 5-fluorouracil, and leucovorin (IFOX) in patients with advanced solid malignancies. Invest New Drugs 2006 ; $24: 117-23$.

47. Kindler HL, Friberg G, Skoog L, et al. Phase I/II trial of gefitinib and oxaliplatin in patients with advanced colorectal cancer. Am J Clin Oncol 2005; $28: 340-4$. 
48. Rothenberg ML, LaFleur B, Levy DE, et al. Randomized phase II trial of the clinical and biological effects of two dose levels of gefitinib in patients with recurrent colorectal adenocarcinoma. J Clin Oncol $2005 ; 23: 9265-74$.

49. Czito BG, Willett CG, Bendell JC, et al. Increased toxicity with gefitinib, capecitabine, and radiation therapy in pancreatic and rectal cancer: phase I trial results. J Clin Oncol 2006; $24: 656-62$.

50. Wolpin BM, Clark JW, Meyerhardt JA, et al. Phase I study of gefitinib plus FOLFIRI in previously untreated patients with metastatic colorectal cancer. Clin Colorectal Cancer 2006 ; $6: 208-13$.

51. Messersmith WA, Laheru DA, Senzer NN, et al. Phase I trial of irinotecan, infusional 5-fluorouracil, and leucovorin (FOLFIRI) with erlotinib (OSI-774): early termination due to increased toxicities. Clin Cancer Res $2004 ; 10: 6522-7$.

52. Townsley CA, Major P, Siu LL, et al. Phase II study of erlotinib (OSI-774) in patients with metastatic colorectal cancer. BrJ Cancer 2006; 94 : 1136-43.

53. Chung KY, Shia J, Kemeny NE, et al. Cetuximab shows activity in colorectal cancer patients with tumors that do not express the epidermal growth factor receptor by immunohistochemistry. J Clin Oncol 2005 ; 23 : 1803-10.

54. Hebbar MA, Wacrenier AB, Desauw CA, et al. Lack of usefulness of epidermal growth factor receptor expression determination for cetuximab therapy in patients with colorectal cancer. Anticancer Drugs $2006 ; 17: 855-7$.

55. Sartore-Bianchi A, Moroni M, Veronese $S$, et al. Epidermal growth factor receptor gene copy number and clinical outcome of metastatic colorectal cancer treated with panitumumab. J Clin Oncol $2007 ; 25: 3238-45$.

56. Cappuzzo F, Hirsch FR, Rossi $\varepsilon$, et al. Epidermal Growth Factor Receptor Gene and Protein and Gefitinib Sensitivity in Non-Small-Cell Lung Cancer. J. Natl. Cancer Inst 2005 ; 97 : 643-55.

57. Khambata-Ford S, Garrett CR, Meropol NJ, et al. Expression of epiregulin and amphiregulin and K-ras mutation status predict disease control in metastatic colorectal cancer patients treated with cetuximab. J Clin Oncol $2007 ; 25: 3230-7$.

58. Lievre A, Bachet JB, Le Corre D, et al. KRAS mutation status is predictive of response to cetuximab therapy in colorectal cancer. Cancer Res $2006 ; 66: 3992-5$.

59. Di Fiore F, Blanchard F, Charbonnier F, et al. Clinical relevance of KRAS mutation detection in metastatic colorectal cancer treated by Cetuximab plus chemotherapy. Br J Cancer 2007 ; 96: 1166-9.

60. Benvenuti S, Sartore-Bianchi A, Di Nicolantonio F, et al. Oncogenic activation of the RAS/RAF signaling pathway impairs the response of metastatic colorectal cancers to anti-epidermal growth factor receptor antibody therapies. Cancer Res 2007 ; $67: 2643-8$.
61. Van Custem $\varepsilon$, Lang I, D'Haens G, et al. KRAS status and efficacy in fisrtline treatment of patients with metastatic colorectal cancer ( $m C R C$ ) treated with FOLFIRI with or without cetuximab: the CRYSTAL experience. ASCO Annual Meeting Proceedings 2008 ; vol. 26, $n^{\circ} 15 S$, Part I of II (abstract 2) : 5 S.

62. Bokemeyer C, Bondarenko I, Hartmann JT, et al. KRAS status and efficacy of fisrt-line treatment of patients with metastatic colorectal cancer ( $M C R C$ ) with FOLFOX with or without cetuximab: the OPUS experience. ASCO Annual Meeting Proceedings 2008 ; vol. 26, $n^{\circ} 15 S$, Part I of II (abstract 4000) : 178s.

63. Tejpar S, Peeters M, Humblet $Y$, et al. Relastionship of efficacy with KRAS status (wild type versus mutant) in patients with irinotecan-refractory metastatic colorectal cancer $(m C R C)$, treated with irinotecan $(q 2 w)$ and escalating doses of cetuximab ( $q 1 \mathrm{w}$ ): the EVEREST experience (preliminary data). ASCO Annual Meeting Proceedings 2008; vol. 26 , $\mathrm{n}^{\circ} 15 \mathrm{~S}$, Part I of II (abstract 4001) : 178s.

64. Saltz LB, Lenz HJ, Kindler HL, et al. Randomized phase II trial of cetuximab, bevacizumab, and irinotecan compared with cetuximab and bevacizumab alone in irinotecan-refractory colorectal cancer: the BOND-2 study. J Clin Oncol 2007 ; 25 : 4557-61.

65. Hecht JR, Mitchell \&P, Chidiac T, et al. Interim results from PACCE: Irinotecan (Iri/bevacizumab (bev) +/- panitumumab (pmab) as firstline treatment $(t x)$ for metastatic colorectal cancer ( $m C R C)$. Proceedings Gastrointestinal Cancers Symposium 2008 ; abstract 279 : 208.

66. Punt CJ, Tol J, Rodenburg A, et al. Randomized phase III study of capecitabine, oxaliplatin and bevacizumab with or without cetuximab in advanced colorectal cancer (ACC), the CAIRO2 study of the Dutch colorectal cancer group (DCCG). ASCO Annual Meeting Proceedings 2008 ; vol. 26, $n^{\circ} 15$ S, Part I of II (abstract LBA4011) : 180s.
TIRÉS À PART

F. Viret 